PROCEEDINGS OF THE

AMERICAN MATHEMATICAL SOCIETY

Volume 125, Number 8, August 1997, Pages 2485-2491

S 0002-9939(97)03869-0

\title{
DECOMPOSITION OF A VECTOR FIELD IN A PARALLELIZED INDUCED BUNDLE
}

\author{
OLEG S. SVIDERSKY
}

(Communicated by Palle E. T. Jorgensen)

\begin{abstract}
The projective method of decomposing of a vector field is applied to constructing a parallelized Lie group action in the framework of Chronometric Theory. An example is considered.
\end{abstract}

\section{INTRODUCTION}

The problem of parallelization arises in the framework of Chronometric Theory from considerations of simplifying the mathematical formulas, but seems to be of interest itself from a mathematical point of view and, from the point of view of physics, for a comparison of different models of the universe.

The general construction of a ("parallelized") induced bundle given in [1] includes:

- a Lie subgroup $N$ of the Lie group $\mathbf{G}$;

- a (local) $C^{\infty}$-action $\phi$ of $\mathbf{G}$ on $N$ which satisfies

$$
\phi(x) y=x y, \text { for } x, y \in N
$$

and

- a finite-dimensional representation $R$ of the subgroup $G_{x_{0}}$ wich fixes a given point $x_{0} \in N$.

Suppose we have a bundle $(\mathbf{G}, H, \mathbf{F})$ over $H$ with the group $\mathbf{G}_{x_{0}}$ acting via $R$ on a finite-dimensional fibre $\mathbf{F}$. Denote by $\mathbf{B}$ the space of $C^{\infty}$-sections of this bundle. We can define the action $\mathbf{U}$ of $G$ on $\mathbf{B}$ by the formula:

$$
\mathbf{U}(g): \boldsymbol{\Psi}(x) \longmapsto S(g, x) \boldsymbol{\Psi}\left(\left(\phi(g)^{-1}\right) x\right),
$$

where $\boldsymbol{\Psi}$ is an arbitrary element of $\mathbf{B}, S(g, x)$ is the $C^{\infty}$-smooth operator function on $\mathbf{G} \times N$ :

$$
S(g, x): \mathbf{F}\left(\phi\left(g^{-1}\right) x\right) \rightarrow \mathbf{F}(x),
$$

$\mathbf{F}(x)$ is the fibre over the point $x \in H$.

We can also define the space $B$ of "parallelized" sections $B=\{\psi(x)\}$, where $\psi(x)=\left(S\left(x x_{0}^{-1}, x\right)\right)^{-1} \mathbf{\Psi}(x)$ and $\psi(x) \in \mathbf{F}\left(x_{0}\right)$ for all $x \in N$.

Theorem 1 below is a variant of Theorem 4.1 of [1] which is adapted to our present purposes.

Received by the editors October 19, 1995 and, in revised form, February 22, 1996.

1991 Mathematics Subject Classification. Primary 22E30.

(C)1997 American Mathematical Society 
Theorem 1. The action $U(g)$ of the group $\boldsymbol{G}$ on $B$ is given by

$$
(U(g) \psi)(x)=R\left(g^{0}\right) \psi\left(\phi\left(g^{-1}\right) x\right), \quad g^{0}=x_{0} x^{-1} g\left(\phi\left(g^{-1}\right)(x)\right) x_{0}^{-1},
$$

and the action of its Lie algebra $\mathcal{G}$ on $B$ is given by

$$
d U(\mathbf{L})=-L+d R(\operatorname{int}(\mathbf{L}))
$$

where $L$ is the horizontal (tangent to $N$ ) component of the vector field on $\boldsymbol{G}$ corresponding to the generator $\boldsymbol{L}$ of $\boldsymbol{G}$, restricted to the group $N$, and int $(\boldsymbol{L})$ is the vertical one.

Thus, if we know both horizontal and vertical components of a vector field $\xi$ corresponding to the generator $\mathbf{L}$ of the Lie group $\mathbf{G}$, we can construct the "parallelized" action of this generator on $B$.

\section{Algorithm}

Let $N$ be a Lie subgroup of a Lie group $G$, let $\phi$ be a $C^{\infty}$-action of $G$ on $N$ such that $\phi(x) y=x y$ for $x, y \in N$, and let $H=G_{e}$ be an isotropy subgroup of the identity element $e \in N$.

Denote by $\mathcal{N}, \mathcal{G}, \mathcal{H}$ the Lie algebras of $N, G$ and $H$ respectively, let $l_{1}, \ldots, l_{k}$ be the generators of Lie group $N$, and let $h_{1}, \ldots, h_{n-k}$ be the generators of Lie group $H$.

Suppose we are given a vector field $\xi(g)$ on $G$. Consider its restriction $\xi(x), x \in$ $N$, on the subgroup $N$. We have

$$
d \mathcal{L}_{x^{-1}}(\xi(x)) \in \mathcal{G},
$$

where $\mathcal{L}_{x^{-1}}$ is the operator of left-multiplying by $x^{-1}$ :

$$
y \mapsto x^{-1} y
$$

on the group $G$.

Write a linear combination $\alpha^{i}(x) l_{i}, i=1, \ldots, k$, where $\alpha^{i} \in C^{\infty}(N)$ are arbitrary smooth functions on $N$, and demand that

$$
d \mathcal{L}_{x^{-1}}(\xi(x))-\alpha^{i}(x) l_{i} \in \mathcal{H}, x \in N .
$$

Theorem 2. The condition (1) determines the functions $\alpha^{i}(x), i=1, \ldots, k$, and

$$
\alpha^{i}(x) l_{i}
$$

is the horizontal component of a vector field $\xi(x)$ and

$$
d \mathcal{L}_{x^{-1}} \xi(x)-\alpha^{i}(x) l_{i}, x \in N,
$$

is the vertical one.

Proof. The proof is based on the evident fact, that the Lie algebra $\mathcal{G}$ (as a vector space) is a direct sum of $\mathcal{N}$ and $\mathcal{H}$ :

$$
\mathcal{G}=\mathcal{N} \oplus \mathcal{H}
$$

Let $l_{1}, \ldots, l_{k}$ be a basis of $\mathcal{N}$, and let $h_{1}, \ldots, h_{n-k}$ be a basis of $\mathcal{H}$. Since

$$
d \mathcal{L}_{x^{-1}} \xi(x) \in \mathcal{G},
$$

there exist functions $\alpha_{1}(x), \ldots, \alpha_{k}(x), \beta_{1}, \ldots, \beta_{n-k}(x)$ such that

$$
d \mathcal{L}_{x^{-1}} \xi(x)=\alpha^{i}(x) l_{i}+\beta^{j}(x) h_{j}, i=1, \ldots, k, j=1, \ldots, n-k,
$$

and this decomposition is unique. 


\section{Applichtion to Chronometric theory}

In this section we define the "oscillator parallelization" of an induced bundle via the following construction.

The Oscillator Lie group $S c$ can be defined as a topologically isomorphic to $\mathbf{R}^{4}$ with the following (nonzero) brackets in its Lie algebra:

$$
\begin{aligned}
& {\left[l_{2}, l_{3}\right]=l_{1},} \\
& {\left[l_{2}, l_{4}\right]=l_{3},} \\
& {\left[l_{3}, l_{4}\right]=-l_{2} .}
\end{aligned}
$$

Consider the following matrices:

$$
\begin{gathered}
l_{1}=\left(\begin{array}{cccc}
2 i & 0 & 0 & 2 i \\
0 & 0 & 0 & 0 \\
0 & 0 & 0 & 0 \\
-2 i & 0 & 0 & -2 i
\end{array}\right), l_{2}=\left(\begin{array}{cccc}
0 & 0 & -1 & 0 \\
0 & 0 & 0 & 0 \\
-1 & 0 & 0 & -1 \\
0 & 0 & 1 & 0
\end{array}\right), \\
l_{3}=\left(\begin{array}{cccc}
0 & 0 & i & 0 \\
0 & 0 & 0 & 0 \\
-i & 0 & 0 & -i \\
0 & 0 & -i & 0
\end{array}\right), \quad l_{4}=\operatorname{diag} \frac{1}{4}(i, i,-3 i, i)
\end{gathered}
$$

which belong to $s u(2,2)$. It's easy to see that their brackets satisfy (2), and hence they generate a Lie subgroup $S c \leq S U(2,2)$ which is isomorphic to the oscillator group.

Define an element $X$ of this subgroup as

$$
\begin{gathered}
X=\exp \left(x_{0} l_{1}+x_{1} l_{2}+x_{2} l_{3}\right) \exp \left(x_{3} l_{4}\right) \\
=e^{i x_{3} / 4}\left(\begin{array}{cccc}
1+\alpha & 0 & -e^{-i x_{3}}\left(x_{1}-i x_{2}\right) & \alpha \\
0 & 1 & 0 & 0 \\
-x_{1}-i x_{2} & 0 & e^{-i x_{3}} & -x_{1}-i x_{2} \\
-\alpha & 0 & e^{i x_{3}}\left(x_{1}-i x_{2}\right) & 1-\alpha
\end{array}\right),
\end{gathered}
$$

where $\alpha=\frac{1}{2}\left(x_{1}^{2}+x_{2}^{2}\right)+2 i x_{0}$, and consider the orbit of the identity element $\mathbf{1} \in \mathbf{U}(2)$ under the action $\phi$ restricted to $S c \leq S U(2,2)$ :

$$
\mathbf{O}_{\mathbf{1}}^{S c}=\left\{\mathbf{x}=\phi(x) \mathbf{1}=(A \mathbf{1}+B)(C \mathbf{1}+D)^{-1}\right\}
$$

where $A, B, C, D$ are $2 \times 2$-blocks of the matrix $X$ :

$$
X=\left(\begin{array}{cc}
A & B \\
C & D
\end{array}\right)
$$

This orbit is

$$
\mathbf{O}_{1}^{S c}=\mathbf{U}(2) \backslash\left\{\left(\begin{array}{cc}
0 & 1 \\
e^{2 i t} & 0
\end{array}\right),-\pi<t \leq \pi\right\},
$$

and the mapping $J: S c \rightarrow \mathbf{U}(2)$ defined by

$$
J(x)=\phi(x) \mathbf{1}
$$

is the one-to-one diffeomorphism between $\mathrm{Sc}$ and $\mathbf{O}_{\mathbf{1}}^{S c}$. (The proofs of these facts are given in the work [2].) Evidently, $J(e)=\mathbf{1}$. 
For the action $\phi_{0}=J^{-1} \phi J$ (where it is defined) we have:

$$
\begin{aligned}
\phi_{0}(x) y & =J^{-1} \phi(x) J(y) \\
& =J^{-1}(\phi(x) \phi(y) \mathbf{1}) \\
& =J^{-1}(\phi(x y) \mathbf{1}) \\
& =J^{-1}(x y) \\
& =x y
\end{aligned}
$$

(since $J^{-1}(x)$ is the solution $y \in S c$ of the equation $J(y)=x$ if it exists).

Notice that, if $h \in S U(2,2)_{1}$ for the action $\phi$, then

$$
\phi_{0}(h) e=J^{-1} \phi(h) J e=J^{-1}(\phi(h) \mathbf{1})=J^{-1}(\mathbf{1})=e,
$$

and $h \in S U(2,2)_{e}$ for the action $\phi_{0}$.

Now we can apply the method described in Section 1 to the following model:

$$
\mathbf{G}=S U(2,2), N=S c, H=(-\mathbf{1}) \mathbf{P}(-\mathbf{1}),
$$

where $\mathbf{P}$ is the scale-extended Poincaré group (being the isotropy subgroup of the point $(-\mathbf{1}) \in \mathbf{U}(2)$ in relation to the action $\phi$, so that $\left(-\mathbf{1}_{\mathbf{4}}\right) \mathbf{P}\left(-\mathbf{1}_{\mathbf{4}}\right)$ with $-\mathbf{1}_{\mathbf{4}}=$ $\left(\begin{array}{cc}-\mathbf{1} & 0 \\ 0 & \mathbf{1}\end{array}\right)$ is the isotropy subgroup of the identity element $\mathbf{1} \in \mathbf{U}(2)$.

In this case:

$$
\begin{gathered}
\xi(x)=\left.\frac{d}{d t}\right|_{t=0}(\exp (t \mathbf{L}) X)=\mathbf{L} X, \\
d \mathcal{L}_{x^{-1}}(\xi(x))=X^{-1} \mathbf{L} X .
\end{gathered}
$$

Lemma 1. If a matrix

$$
\zeta=\left(\begin{array}{ll}
a & b \\
c & d
\end{array}\right)
$$

satisfies the condition

$$
a+b=c+d,
$$

where $a, b, c, d$ are its $(2 \times 2)$-blocks, then $\zeta \in \mathbf{G}_{e}$.

Proof. A matrix $g=\left(\begin{array}{cc}A & B \\ C & D\end{array}\right)$ belongs to $\mathbf{P}$ if its $(2 \times 2)$-blocks $A, B, C, D$ satisfy the condition $A-B=C-D$, or, in other words, the matrix $\Omega^{-1} g \Omega$, where $\Omega=\left(\begin{array}{cc}\mathbf{1} & -\mathbf{1} \\ \mathbf{1} & \mathbf{1}\end{array}\right)$, has the form

$$
\left(\begin{array}{cc}
A^{\prime} & B^{\prime} \\
0 & C^{\prime}
\end{array}\right) .
$$

Let the matrix $\zeta$ satisfies the condition (4). Then the matrix

$$
\Omega^{-1}(-\mathbf{1}) \zeta(-\mathbf{1}) \Omega
$$

has the form (5), and the matrix

$$
\exp \left(\Omega^{-1}(\mathbf{- 1}) \zeta(-\mathbf{1}) \Omega\right)
$$

has the same form and, consequently, belongs to $\mathbf{P}$, and $\zeta \in \mathbf{G}_{e}$. 


\section{APPENDIX}

In Table 1 the vector fields on the Lie group Sc corresponding to the generators of $S U(2,2)$ via the action $\phi_{0}$ are listed. We denote $s=\sin x_{3}, c=\cos x_{3}$.

We don't list the internal parts of the generators since their formulas are overly bulky.

\section{TABLE 1}

\begin{tabular}{|c|l|}
\hline $\begin{array}{c}\text { Generator of } \\
\mathrm{SU}(2,2)\end{array}$ & Vector field on Sc \\
& $L=\alpha_{1} l_{1}+\alpha_{2} l_{2}+\alpha_{3} l_{3}+\alpha_{4} l_{4}$ \\
\hline \hline $\mathbf{L}_{-10}$ & $\alpha_{1}=\frac{1}{2}+\frac{1}{8}\left(x_{1}^{2}+x_{2}^{2}\right)^{2}+2 x_{0}^{2}$ \\
& $\alpha_{2}=\frac{1}{2}\left(x_{1}^{2}+x_{2}^{2}\right)\left(x_{2} c+x_{1} s\right)+2 x_{0}\left(x_{1} c-x_{2} s-1\right)$ \\
& $\alpha_{3}=-1+\frac{1}{2}\left(x_{1}^{2}+x_{2}^{2}\right)\left(x_{2} s-x_{1} c+1\right)+2 x_{0}\left(x_{2} c+x_{1} s\right)$ \\
& $\alpha_{4}=2-\left(x_{1}^{2}+x_{2}^{2}\right)+2\left(x_{1} c-x_{2} s\right)$
\end{tabular}




\begin{tabular}{|c|c|}
\hline $\begin{array}{l}\text { Generator of } \\
\mathrm{SU}(2,2)\end{array}$ & $\begin{array}{l}\text { Vector field on Sc } \\
L=\alpha_{1} l_{1}+\alpha_{2} l_{2}+\alpha_{3} l_{3}+\alpha_{4} l_{4}\end{array}$ \\
\hline $\mathbf{L}_{03}$ & $\begin{aligned} \alpha_{1}= & -x_{0}\left(1+x_{1}\right)+\frac{1}{4} x_{2}\left(x_{1}^{2}+x_{2}^{2}\right) \\
\alpha_{2}= & \frac{1}{4}\left(x_{1}^{2}+x_{2}^{2}\right)-\frac{1}{2}\left(x_{1} c-x_{2} s-x_{1}\right)-\frac{1}{4}\left(x_{1}^{2}-3 x_{2}^{2}\right) c \\
\quad & \quad-\left(x_{0}-x_{1} x_{2}\right) s \\
\alpha_{3}= & x_{0}-\frac{1}{2}\left(x_{2} c-x_{1} s-x_{2}\right) \\
& \quad-\frac{1}{4}\left(x_{1}^{2}-3 x_{2}^{2}\right) s+\left(x_{0}-x_{1} x_{2}\right) c \\
\alpha_{4}= & x_{2}-c+x_{1} s+x_{2} c\end{aligned}$ \\
\hline $\mathbf{L}_{12}$ & $\begin{aligned} \alpha_{1}= & \frac{1}{8}\left(x_{1}^{2}+x_{2}^{2}\right)^{2}-\frac{1}{2}\left(x_{1}^{2}+x_{2}^{2}\right)+2 x_{0} \\
\alpha_{2}= & \frac{1}{2}\left(x_{1}^{2}+x_{2}^{2}-2\right)\left(x_{2} c+x_{1} s\right)+2 x_{0}\left(x_{1} c-x_{2} s-1\right) \\
\alpha_{3}= & \frac{1}{2}\left(x_{1}^{2}+x_{2}^{2}\right)\left(1-x_{1} c+x_{2} s\right) \\
& \quad+x_{1} c-x_{2} s+2 x_{0}\left(x_{2} c+x_{1} s\right) \\
\alpha_{4}= & \left(x_{1}^{2}+x_{2}^{2}\right)-2 x_{1} c+2 x_{2} s\end{aligned}$ \\
\hline $\mathbf{L}_{23}$ & $\begin{aligned} \alpha_{1}= & \frac{1}{4}\left(x_{1}^{2}+x_{2}^{2}\right)-\frac{1}{4} x_{1}\left(x_{1}^{2}+x_{2}^{2}-2\right)-x_{0} x_{2} \\
\alpha_{2}= & x_{0}+\frac{1}{2}\left(x_{2}+s\right)+\frac{1}{2}\left(x_{2} c+x_{1} s\right) \\
& \quad-\left(x_{0}+x_{1} x_{2}\right) c-\frac{1}{4}\left(3 x_{1}^{2}-x_{2}^{2}\right) s \\
\alpha_{3}= & -\frac{1}{4}\left(x_{1}^{2}+x_{2}^{2}\right)-\frac{1}{2}\left(\left(1+x_{1}\right) c-x_{2} s-x_{1}\right)+\frac{1}{4}\left(3 x_{1}^{2}-x_{2}^{2}\right) c \\
& \quad-\left(x_{0}-x_{1} x_{2}\right) s \\
\alpha_{4}= & \left(1+x_{1}\right)(c-1)-x_{2} s\end{aligned}$ \\
\hline $\mathbf{L}_{31}$ & $\begin{aligned} \alpha_{1}= & -x_{0}-\frac{1}{2} x_{2}+\frac{1}{4} x_{2}\left(x_{1}^{2}+x_{2}^{2}\right)-x_{0} x_{1} \\
\alpha_{2}= & \frac{1}{2}\left(1+x_{2}-x_{1} c+x_{2} s\right)+\frac{1}{4}\left(3 x_{2}^{2}-x_{1}^{2}\right) c-\left(x_{0}-x_{1} x_{2}\right) s \\
& \quad+\frac{1}{4}\left(x_{1}^{2}+x_{2}^{2}\right) \\
\alpha_{3}= & x_{0}-\frac{1}{2}\left(x_{2} c+x_{1} s-x_{2}+s\right) \\
& \quad+\frac{1}{4}\left(3 x_{2}^{2}-x_{1}^{2}\right) s+\left(x_{0}-x_{1} x_{2}\right) c \\
\alpha_{4}= & s+x_{2}+x_{1} s+x_{2} c\end{aligned}$ \\
\hline $\mathbf{L}_{04}$ & $\begin{aligned} \alpha_{1}= & \frac{1}{2}-\frac{1}{4}\left(1+x_{1}\right)\left(x_{1}^{2}+x_{2}^{2}\right)-x_{0} x_{2} \\
\alpha_{2}= & -x_{0}-\frac{1}{2}\left(x_{2}(c-1)+x_{1} s\right) \\
& \quad-\left(x_{0}+x_{1} x_{2}\right) c-\frac{1}{4}\left(3 x_{1}^{2}-x_{2}^{2}\right) s \\
\alpha_{3}= & \frac{1}{4}\left(x_{1}^{2}+x_{2}^{2}\right)+\frac{1}{2}\left(x_{1}(c-1)-x_{2} s-1\right) \\
& +\frac{1}{4}\left(3 x_{1}^{2}-x_{2}^{2}\right) c-\left(x_{0}+x_{1} x_{2}\right) s \\
\alpha_{4}= & 1+c-\left(x_{1}(c+1)-x_{2} s\right)\end{aligned}$ \\
\hline $\mathbf{L}_{14}$ & $\begin{aligned} \alpha_{1}= & \frac{1}{4}\left(x_{1}^{2}+x_{2}^{2}\right)+\frac{1}{4} x_{1}\left(x_{1}^{2}+x_{2}^{2}-2\right)+x_{0} x_{2} \\
\alpha_{2}= & x_{0}+\frac{1}{2}\left(x_{2}(c-1)+\left(x_{1}-1\right) s\right) \\
& \quad+\frac{1}{4}\left(3 x_{1}^{2}-x_{2}^{2}\right) s+\left(x_{0}+x_{1} x_{2}\right) c \\
\alpha_{3}= & \frac{1}{2}\left(1+x_{1}+\left(1-x_{1}\right) c+x_{2} s\right) \\
& \quad-\frac{1}{4}\left(3 x_{1}^{2}-x_{2}^{2}\right) c+\left(x_{0}+x_{1} x_{2}\right) s \\
\alpha_{4}= & -1-c+x_{1}(c-1)-x_{2} s\end{aligned}$ \\
\hline $\mathbf{L}_{24}$ & $\begin{aligned} \alpha_{1}= & -x_{0}+x_{0} x_{1}-\frac{1}{4} x_{2}\left(x_{1}^{2}+x_{2}^{2}-2\right) \\
\alpha_{2}= & \frac{1}{2}\left(1-x_{1}\right)+\frac{1}{4}\left(x_{1}^{2}+x_{2}^{2}\right)+\frac{1}{4}\left(x_{1}^{2}-3 x_{2}^{2}+2\right) c+ \\
& \quad\left(x_{0}-x_{1} x_{2}\right) s-\frac{1}{2}\left(x_{1} c-x_{2} s\right) \\
\alpha_{3}= & x_{0}-\frac{1}{2}\left(x_{2}(c+1)+\left(x_{1}-1\right) s\right) \\
& \quad+\frac{1}{4}\left(x_{1}^{2}-3 x_{2}^{2}\right) s-\left(x_{0}-x_{1} x_{2}\right) c \\
\alpha_{4}= & x_{2}(c-1)+\left(x_{1}-1\right) s\end{aligned}$ \\
\hline $\mathbf{L}_{34}$ & $\begin{array}{l}\alpha_{1}=-\frac{1}{2}+\frac{1}{2}\left(x_{1}^{2}+x_{2}^{2}\right) \\
\alpha_{2}=x_{2} c+x_{1} s \\
\alpha_{3}=1-x_{1} c+x_{2} s \\
\alpha_{4}=0\end{array}$ \\
\hline
\end{tabular}


Note. From the equations

$$
\begin{gathered}
l_{i} x_{j}=\left.\frac{d}{d t}\right|_{t=0} x_{j}\left(\exp \left(t l_{i}\right) X\right), \\
i=1, \ldots, 4,
\end{gathered}
$$

we can find the action of the vector fields $l_{1}, \ldots, l_{4}$ on the coordinate functions $x_{0}, \ldots, x_{3}$ and, consequently, represent them as the linear combinations of the basic vector fields $\partial_{0}, \ldots, \partial_{3}$. We'll have:

$$
\begin{aligned}
& l_{1}=\partial_{0}, \\
& l_{2}=-\frac{1}{2} x_{2} \partial_{0}+\partial_{1}, \\
& l_{3}=\frac{1}{2} x_{1} \partial_{0}+\partial_{2}, \\
& l_{4}=-x_{2} \partial_{1}+x_{1} \partial_{2}+\partial_{3},
\end{aligned}
$$

and, for example,

$$
\begin{gathered}
L_{34}=\frac{1}{2}\left(-1+x_{1}+\left(x_{1}^{2}+x_{2}^{2}\right)(1-c)\right) \partial_{0}+\left(x_{2} c+x_{1} s\right) \partial_{1}+\left(1-x_{1} c+x_{2} s\right) \partial_{2} . \\
\text { REFERENCES }
\end{gathered}
$$

1. Paneitz S.M., Segal I.E., Analysis in in space-time bundles. I, J. Funct. Anal. 1982. Vol. 47, pp. 78-142. MR 83k:22042

2. Svidersky O.S., "Oscillator Parallelization of the Scalar Induced Bundle", Sibirsky Matematichesky Zhurnal, Vol. 36, No. 5(213), 1995., pp. 1122-1229.

Department of Mathematics, University of Iowa, Iowa City, Iowa 52242-1419

E-mail address: svidersk@math.uiowa.edu 